Tune B. Bonné

Karin Lüdtke

Rainer Jordan

Petr Štěpánek

Christine M. Papadakis

\section{Aggregation behavior of amphiphilic poly(2-alkyl-2-oxazoline) diblock copolymers in aqueous solution studied by fluorescence correlation spectroscopy}

Published online: 15 September 2004

(c) Springer-Verlag 2004

The online version of the original article can be found at http://dx.doi.org/10.1007/ s00396-004-1131-2

T. B. Bonné · C. M. Papadakis $(\bowtie)$

Fakultät für Physik und

Geowissenschaften, Universität Leipzig,

Linnéstr. 5, 04103 Leipzig, Germany

E-mail: Christine.Papadakis@ph.tum.de

K. Lüdtke $\cdot$ R. Jordan

Chemie Department,

Technische Universität München,

Lichtenbergstr. 4, 85747 Garching,

Germany

P. Štěpánek

Institute of Macromolecular Chemistry,

Academy of Sciences of the Czech

Republic, Heyrovsky Sq. 2,

CZ-16206 Prague, Czech Republic
Present address: T. B. Bonné

C. M. Papadakis

Physik Department E13,

Technische Universität München,

James-Franck-Str. 1, 85747 Garching,

Germany

R. Jordan

Department of Chemistry,

Chemical Engineering and Materials

Science, Polytechnic University,

Six Metrotech Center, Brooklyn,

NY 11201, USA

Table 1 Analytical values of the polymers

\begin{tabular}{|c|c|c|c|c|c|c|c|}
\hline Polymer & $\begin{array}{l}\mathrm{DP}_{\mathrm{n}} \\
{[\mathrm{M} 1]_{0} /[\mathrm{I}]_{0}} \\
1 \text { st block }\end{array}$ & $\begin{array}{l}\mathrm{DP}_{\mathrm{n}} \\
{[\mathrm{M} 2]_{0} /[1]_{0}} \\
\text { 2nd block }\end{array}$ & $\begin{array}{l}\text { DP } \\
\text { 1st block }\end{array}$ & $\begin{array}{l}\text { DP } \\
\text { 2nd block }\end{array}$ & $\begin{array}{l}\mathrm{M}_{\mathrm{n}} \\
{ }^{1} \mathrm{H}-\mathrm{NMR}^{\mathrm{c}}\end{array}$ & Yield $/ \%{ }^{\mathrm{d}}$ & $\mathrm{PDI}^{\mathrm{e}}$ \\
\hline $\mathrm{P}\left[(\mathrm{MOx})_{40}(\mathrm{NOx})_{7}\right]$ & 30 & 6 & 40 & 7 & 4034 & 89 & 1.20 \\
\hline $\mathrm{P}\left[(\mathrm{NOx})_{10}(\mathrm{MOx})_{32}\right]$ & 7 & 40 & 10 & 32 & 4796 & 96 & 1.07 \\
\hline $\mathrm{PMOX}_{26}$ & 30 & - & 26 & - & 2313 & 87 & 1.21 \\
\hline
\end{tabular}

${ }^{a}$ Degree of polymerization $\left(\mathrm{DP}_{\mathrm{n}}\right)$ calculated from initial monomer/initiator feed

${ }^{b}$ Degree of polymerization calculated from ${ }^{1} \mathrm{H}-\mathrm{NMR}$ spectra (from end group analysis)

${ }^{\mathrm{c}}$ Molar mass from ${ }^{1} \mathrm{H}-\mathrm{NMR}$ spectra

${ }^{\mathrm{d}}$ Yield vs initial monomer feed

${ }^{\text {e}}$ Polydispersity index (PDI: $\bar{M}_{w} / \bar{M}_{n}$ ) as measured by GPC 